\title{
Effects of orientation and contrast upon targets in straight and curved arrays
}

MS 7237_AG

\author{
Michael W. Levine ${ }^{1,2}$ \\ Jennifer E. Anderson ${ }^{1}$ \\ J. Jason McAnany ${ }^{1,3}$
}

University of Illinois at Chicago

${ }^{1}$ Department of Psychology

${ }^{2}$ Laboratory of Integrative Neuroscience

${ }^{3}$ Department of Ophthalmology and Visual Sciences

Corresponding Author:

Dr. Michael Levine

Department of Psychology, M/C 285

1007 West Harrison Street

Chicago, IL 60607-7137

USA

Email: $\quad$ Mikel@uic.edu

Phone: $\quad 1-312-996-6133$

Fax: $\quad 1-312-413-4122$

Key words: Grid illusions, blanking, vanishing disk, extinction, curved grids, orientation Running head: Orientation of targets in grids 


\begin{abstract}
A regular array of black squares against a gray background increases the threshold for a light disk in an intersection of the alleys separating the squares, an illusion variously called the extinction illusion, "blanking" phenomenon, or "vanishing disk". Curving the alleys (making the squares into curved "tetragons") further increases the thresholds for both light and dark disks. This raises three questions: (1) Do the edges of the tetragons interact with similarly oriented components of a target? (2) Is blanking a different phenomenon from the obscuring (more moderate increase in threshold in the presence of an array) that affects dark disks, or is blanking simply weaker for dark disks? (3) If blanking and obscuring are different effects, is blanking a function of light versus dark targets or dependent upon contrast relative to the polarity of the inducing array? We replaced the target disks with parallel line segments to explore the influence of orientation of the line segments relative to the nearby orientation of the alleys. We show that the blanking phenomenon is sensitive to the orientation of the line segments, while the weaker obscuring of dark targets is not. We also examine these effects with white tetragons. Reversing the polarity of the tetragons exchanged which line segments were orientation-sensitive, although the effectiveness of white tetragons for blanking was weaker than that of black tetragons. We consider possible reasons why white tetragons may be less effective than black tetragons under our conditions.
\end{abstract}




\section{Introduction}

The mechanisms of the grid illusions have been the subject of considerable research. In their canonical form, the grid illusions consist of a regular array of black squares separated by lighter alleys. The Hermann grid has white alleys, and illusory dark "smudges" are seen in intersections of the alleys peripheral to fixation (Fig. 1A); the scintillating grid has gray alleys with white disks in the intersections, and transient black dots appear centered upon disks away from the center of gaze as the eyes scan the grid (Fig. 1B). These illusions are also effective in negative images, with white squares on darker alleys. Note that the illusions are the appearance of features not physically present. The explanation for the illusory effects are not entirely understood, as the traditional lateral inhibition explanation for the Hermann illusion, proposed by Hering (1890) and elaborated by Baumgartner (1960), has proven inadequate (Geier et al., 2004; de Lafuente and Ruiz, 2004; Schiller and Carvey, 2005; Geier et al., 2008; Bach, 2009).

Two additional grid illusions with face similarities to the scintillating grid are illusions of disappearance; in these, a normally suprathreshold target is not detected when placed in an intersection of the alleys. The "extinction illusion" was introduced by Ninio and Stevens (2000) and examined by Araragi and Kitaoka (2011). In the extinction illusion, the intersections all contain white disks with black outline rings; many of the outlined disks disappear (Fig. 1C). This illusion also is evident in negative images (Ninio and Stevens, 2000).

A variant of the extinction illusion is called the "blanking" phenomenon or "vanishing disk" (McAnany and Levine, 2004, 2005; Howe and Livingstone, 2007; Levine and McAnany, 2008). In the blanking phenomenon, a single light target disk (without the black ring) is shown in the intersection of gray alleys in a regular array of black squares. High contrast is needed for the disk to be detectable (Fig. 1D). This effect may be seen in the figure by fixating on 


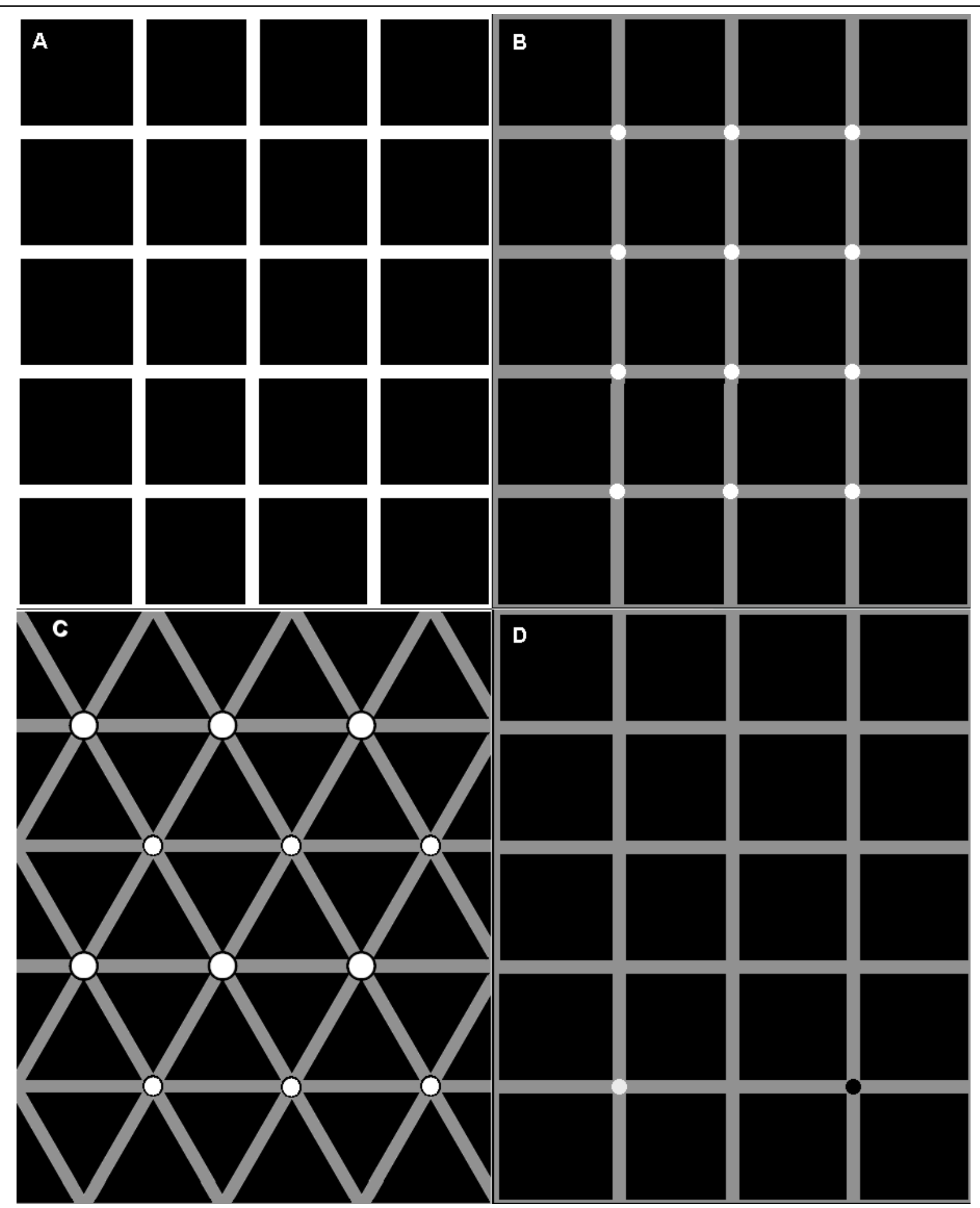

Figure 1: Four grid illusions. A. Hermann grid. B. Scintillating grid C. The extinction illusion. D. Blanking (vanishing disk; light disk) and obscuring (dark disk). 
one of the upper intersections in part B and noting the disappearance of the white target disk in the lower left intersection in part D (image size, illumination, and viewing distance may affect the effectiveness of these demonstrations). The vanishing disk illusion was demonstrated with brief exposures that preclude Troxler fading (McAnany and Levine, 2005; Levine and McAnany, 2008), but has hitherto not been tested with negative images.

There are also somewhat elevated thresholds for dark disks that presumably are not subject to blanking, as well as for the extremely elevated thresholds for the light disks that are subject to it (McAnany and Levine, 2004). That is, dark disks in the intersections had higher thresholds than the same disks against a field of uniform gray equal to that of the alleys. This may be seen by noting the possible weak disappearance of the black disk in Fig. 1D when fixating on an intersection in part B. Because the effect upon dark disks was so much less dramatic than the effect upon light disks, and because arrays of black tetragons produce only dark illusions in the Hermann or scintillating grids, it was suggested that an obscuring effect raises thresholds for the dark disks (Levine and McAnany, 2008). In essence, obscuring would affect any target in a grid, but blanking would affect only the light disks..

Thresholds to both light and dark disks were increased further when the alleys were curved, even though this resulted in minimal change in the area of the alleys (Levine and McAnany, 2008). The effect of curvature is associated with the increased complexity of grids with curved alleys. Complexity could be quantified either by the information content of the grid, or by the power in the Fourier spectrum of the grid (Levine and McAnany, 2008). Curved alleys introduce components at varied orientations. Since disks are homogeneous in orientation, there is no way to say whether orientation of alley edges is a factor, or curvature simply increases complexity. 
Although the blanking phenomenon and obscuring effect do not necessarily invoke the same mechanisms as the Hermann grid or scintillating grid despite the similarity of their configurations, they are useful for investigation. The threshold for the detection of a target can be measured quantitatively by forced choice methods, without requiring a subjective evaluation by the observer. The subjects cannot easily manufacture an effect based on preconceptions. We have exploited this advantage to demonstrate that blanking is due in part to peripheral mechanisms and in part to central mechanisms; blanking is present but much weaker when the disk and grid are presented dichoptically than when presented to the same eye (McAnany and Levine, 2005). We further showed that blanking is stronger (detection thresholds for the disks are higher) when the alleys are curved (Levine and McAnany, 2008).

In the present study, we address three questions. First, do the oriented components of the curved alleys interact with similarly oriented components of a target? Disks comprise all orientations equally, so we replaced the target disk with a pair of parallel line segments (see Fig. 2A). Although two short parallel line segments are not devoid of components at orientations other than the orientation of the line segments, that orientation dominates. We used line segments parallel to the major directions of the alleys (vertical and horizontal, which we refer to as “cardinal"), and line segments at $45^{\circ}$ to vertical (“diagonal” line segments). To avoid the complications of Troxler fading and eye movements, we used only brief presentations of the stimuli.

Light line segments, which are subject to the blanking phenomenon, showed orientation selectivity; that is, thresholds for light line segments depended upon their orientation relative to the nearby alleys. Dark line segments did not show this orientation dependence, with all orientations displaying equal thresholds within any particular array of tetragons ${ }^{1}$ (experiment 1 ). 
These results also address a second question: Is blanking a different phenomenon from the obscuring that affects dark disks, or is the blanking phenomenon simply weaker for dark disks? The finding that orientation affects only the light line segments (which are subject to blanking) is consistent with our suggestion that blanking and obscuring (by complexity) are separate phenomena.

The final question is whether blanking, present for light line segments but not for dark ones in an array of black tetragons, is a function of light versus dark targets, or depends upon contrast relative to the polarity of the inducing array. That is, would the orientation effect apply instead to dark line segments if the array comprised white tetragons instead of black ones? Blanking is weaker with white tetragons, such that the thresholds for light and dark line segments are nearly equal; however, the presence or absence of an orientation effect served as a diagnostic to indicate that blanking is evinced by contrast relative to the array, not simply light versus dark (experiment 2).

As an additional control that the lack of orientation specificity in the obscuring effect is not simply that orientation cannot be detected at the lower contrasts, we repeated the experiments asking the subjects to determine orientation of the line segments. The thresholds for determining orientation followed the same pattern as those for detecting the location of the line segments.

\section{Material and Methods}

\subsection{Subjects}

Six subjects ( 2 males and 3 females, aged 24 to 31 plus one male aged 67; with normal or corrected-to-normal vision) participated in these experiments. Subjects included the three authors; all subjects participated in all of the experiments. An additional naïve subject 
participated in only the first experiment before declining to continue; to ensure fair comparisons across experiments his data were excluded from the figures and analyses, although they were completely consistent with the other subjects' data from that experiment.

\subsection{Display}

The equipment and general methods have been described elsewhere (Levine and McAnany, 2008). Briefly, the subject was seated $35 \mathrm{~cm}$ from an EIZO 19" FlexScan FX-D7 display monitor (1024 X 768 pixels, $85 \mathrm{~Hz}$ refresh rate, 256 gray levels), which supplied the only illumination during the experiments. Chin and forehead rests maintained head position. The procedures were approved by a University of Illinois at Chicago Institutional Review Board.

The stimulus display consisted of two arrays of eight tetragons each (see Fig. 2A). The arrays were separated vertically by $8.4^{\circ}$; the separation was $6.3^{\circ}$ for one of the authors, who was unable to detect the target stimuli at the larger eccentricity. The target line segments were placed at random in either the upper or lower array, and could be in either the left or right intersection $\left(4.7^{\circ}\right.$ left or right of the vertical centerline). Alleys could be straight or curved according to a sinusoid; curvature is expressed by the amplitude of the sine as a percentage of its period, which was the distance from intersection center to intersection center. These arrays of tetragons are identical to those we used previously (Levine and McAnany, 2008).

Following each $235 \mathrm{~ms}$ presentation of the array of tetragons and target, the display was replaced by a response screen having a central circle surrounded by a halo of smaller circles at a radius of $11^{\circ}$ (Fig. 2B or C). The subject clicked one of the smaller circles to indicate his or her response.

There were four possible target line segment orientations: vertical and horizontal, which we refer to as "cardinal" as shown in the upper row of intersections in Fig. 2A, or right- and left- 


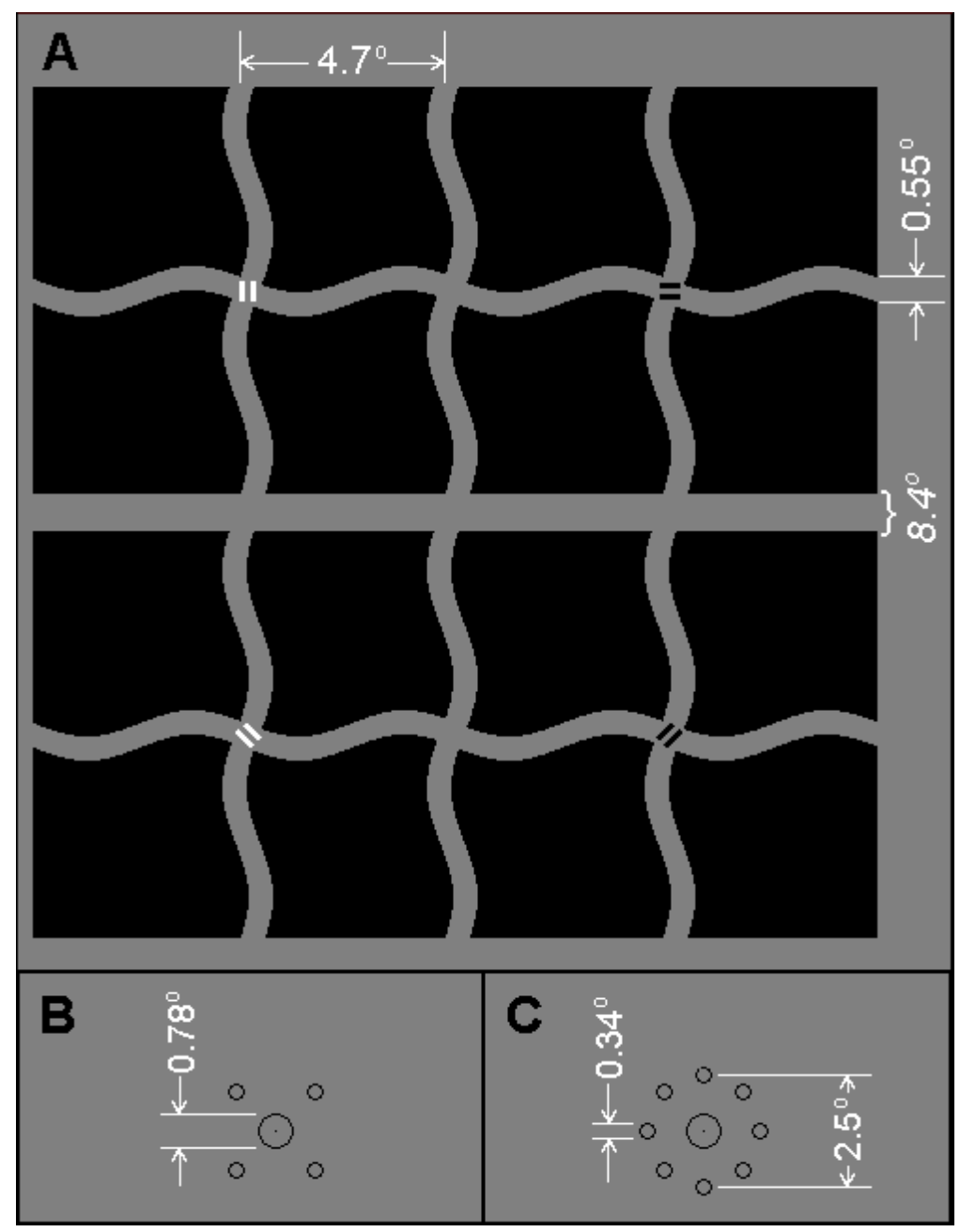

Figure 2: Stimuli used in the experiments. Chimera of screen shots. A. Representation of the display; in practice, the upper and lower block of tetragons was separated by $8.4^{\circ}$ (6.3 $3^{\circ}$ for one subject), and a pair of line segments was present in only one of the four lateral intersections.

Curvature shown is $6.3 \%$. Cardinal orientations are in the upper grid, diagonal in the lower; light line segments on the left and dark on the right. B. Response pattern for experiments 1 and 2 . The central dot was placed where the small fixation circle would be before presentation of the grid. C. Response pattern for experiment 3 . It is the same as the pattern in B except for the addition of circles at the top, bottom, left, and right. leaning $\left(45^{\circ}\right.$ from vertical),

referred to as "diagonal" as

shown in the lower row. There

were three possible alley

curvatures: $0 \%, 2.7 \%$ and

$6.3 \%$. The cardinal line

segments aligned with the

alleys at $0 \%$. The $6.3 \%$

curvature produced alleys with

an angle to the cardinal line

segments of about $20^{\circ}$,

approximately $40 \%$ of the

difference between the

orientations of the cardinal and

diagonal target line segments.

Each run comprised

four randomly interlaced

staircases. The four staircases

in a run represented the four

possible target line segment

orientations. Each run included

only one of the three possible

curvatures, and presented only light (left column in Fig.1A) or only dark (right column) line 
segments. A single session consisted of seven runs (three curvatures with dark or light line segments, plus one control run). The control run consisted of line segments with no array of tetragons (just line segments in the appropriate positions on the gray background). The control could be either dark or light line segments, with one set tested within a session of black tetragons and the other set tested within a session of white tetragons. A session typically lasted about 50 minutes. The order of runs was randomized for each subject.

Before the first presentation in a run, the subject adapted to the mean gray of the screen $\left(24 \mathrm{~cd} / \mathrm{m}^{2}\right)$ for $30 \mathrm{sec}$. The mean gray was the same as the alleys in the array of tetragons and the background of the response screen. The maximum available luminance (white) was $92 \mathrm{~cd} / \mathrm{m}^{2}$.

Responses were made by clicking the appropriate circle on the response pattern (Fig. 2B or C). In experiments 1 and 2, the pattern in Fig. 2B would appear; if the line segments were perceived in the upper right intersection, the correct response would be the circle at 1:30; lower right would be represented at 4:30, and so forth. In experiment 3, Fig. $2 \mathrm{C}$ appeared; if the line segments were perceived to be vertical, the correct response would be the circle at 12:00 or the circle at 6:00; if tilted to the left, the response would be 4:30 or 10:30, and so forth. A mouse icon consisting of a broad arrow comprising all four orientations (Microsoft arrow ARW10NW.ico) was visible only when a response was requested. If the response was ambiguous, the response pattern persisted and the subject was cued (by a beep) to try again; when the response was acknowledged the response pattern was replaced by a single small circle in the same central location, and the next trial began $250 \mathrm{~ms}$ later.

\subsection{Staircase method for determining threshold}

Thresholds were determined by a staircase procedure. Four staircases were interlaced at random within each run; each staircase presented one of the possible orientations of the line segments. 
The subject selected the intersection in which the line segments appeared (experiments 1 and 2) or the orientation of the line segments (experiment 3). Because the task was a randomized four alternative forced choice, there was no influence of expectation, and no bias was conferred. No feedback was offered.

Two consecutive correct responses in a particular staircase resulted in a decrease in contrast of the target (line segment pair) on its next presentation in that staircase by $0.36 \mathrm{~cd} / \mathrm{m}^{2}$; an incorrect response increased the contrast by twice as much as the decrement step. A run continued until there were at least 16 reversals in each of the four concurrent staircases. Although staircases and positions were chosen at random, the probability of testing a staircase that had completed 16 or more reversals was slightly reduced.

Threshold was taken as the average of the luminances at the final six reversals. Threshold luminances were converted to Weber contrast by dividing the difference between target luminance and alley luminance by the alley luminance. Absolute values were used so dark and light targets could be compared within a particular array. Thresholds for vertical and horizontal target line segments were averaged and presented as "cardinal" thresholds; left- and right-leaning thresholds were averaged and presented as "diagonal" thresholds.

\section{Experiments and Results}

\subsection{Experiment 1: Detection in arrays of black tetragons}

This experiment tested whether detection of line segments would be affected by sharing orientation components with tetragons in the array. Would detection of a target in an intersection be influenced by that target's orientation? Would any effect of orientation be manifested for dark targets that are presumably not affected by the blanking phenomenon? 
Light line segments, which should be difficult to perceive because of the blanking phenomenon, did indeed produce high thresholds for all subjects. What was most noticeable in the data from each subject was that the cardinal line segments had a higher threshold than the diagonal line segments at $0 \%$ curvature. That threshold level remained nearly constant for light cardinal line segments as curvature was increased. The thresholds for light diagonal line segments increased with curvature, in many cases exceeding that for light cardinal line segments at the highest curvature.

Dark line segments, on the other hand, showed no such orientation dependence. In most cases, the thresholds for dark cardinal line segments and dark diagonal line segments were nearly identical, with thresholds for each rising slightly in parallel with increased curvature.

These results are summarized in Fig. 3, which shows the average threshold contrasts for each line segment type (cardinal or diagonal, light or dark) across all subjects. Each of the subjects showed a pattern consistent with this average. A paired $t$-test for the difference in threshold between cardinal and diagonal line segments was significant for light line segments at $0 \%$ curvature $(t[11]=5.52, p<0.001)$; no other difference between cardinal and diagonal

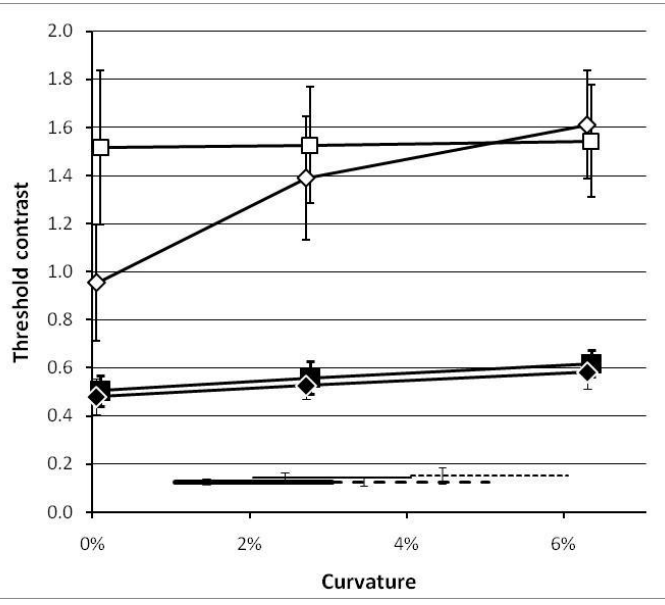

Figure 3: Results of experiment 1. Threshold contrast as a function of alley curvature. Subject indicated which intersection contained the line segments. Black tetragons. Averages of all subjects. Open symbols represent light line segments, closed symbols represent dark line segments; squares represent cardinal line segments, diamonds represent diagonal line segments. The horizontal lines near the bottom represent targets in the absence of a grid: heavy solid = dark cardinal, long dashed = dark diagonal; thin solid = light cardinal, short dashed = light diagonal. Curves are slightly shifted horizontally to segregate error bars. Error bars indicate $95 \%$ confidence intervals. 
line segments (in the same array of tetragons) was significant.

Thresholds for dark line segments were dramatically lower than those for light line segments, consistent with lack of the blanking effect. In the absence of an array of tetragons, thresholds for line segments of any orientation, light or dark, were all essentially equal and considerably lower than for dark line segments in an array. The higher threshold for dark line segments in the array we attribute to the obscuring phenomenon.

The effects of orientation are highlighted in Fig. 4, where the thresholds for diagonal light line segments were subtracted from the thresholds for cardinal light line segments at each curvature (by subject). $95 \%$ confidence intervals show a significant difference at $0 \%$ curvature (that is, the error bars do not extend to zero) and not at any other curvature (curvature scale at the top of the figure, running right to left). A similar comparison of cardinal and diagonal dark line segments indicates no significant difference at any curvature.

Figure 4 also indicates that the differences are related specifically to the angles at which the tetragon edges depart from the intersections. The abscissa represents the difference in angle between the target line segments and the nearest tetragon edges (since the vertical and horizontal alleys are orthogonal

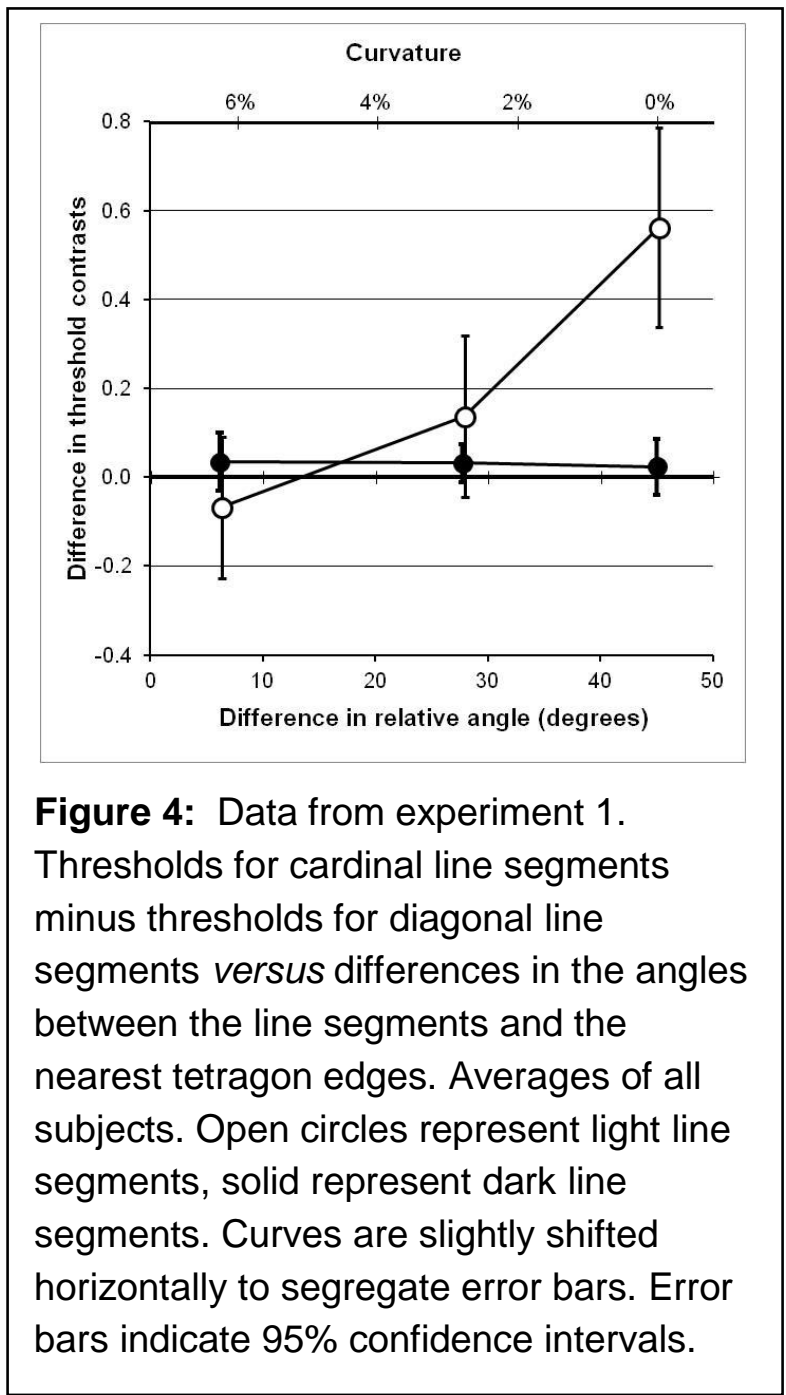


as they depart an intersection, the same difference in angle applies to both vertical and horizontal line segments; similarly, left- and right-leaning line segments share an angle). Light line segments display a nearly linear monotonic relationship with the local edge orientations, while dark line segments show no relationship. (Note that the largest differences in angle are at the lowest curvatures; 0\% curvature represents the largest differences between target and tetragons, at the right in this graph.)

\subsection{Experiment 2: Effect of white tetragons}

We have assumed that light line segments are subject to the blanking phenomenon and dark line segments are not because the tetragons are black (McAnany and Levine, 2004). The second experiment was designed to test that assumption.

The assumption was based on the considerably higher contrast threshold for light stimuli than for dark in the presence of black tetragons, and the observations that Hermann grids and scintillating grids with white squares produce illusions of the opposite contrast (light smudges and white scintillations). But perhaps the significant difference in the blanking phenomenon is light versus dark, and not whether the stimulus is of opposite contrast to the tetragons. That is, is blanking a result of an actual illusion, or a difference between processing by ON and OFF systems? In this experiment, we use white tetragons instead of black. If the illusion depends on contrast relative to the tetragons, we should expect dark line segments to show orientation selectivity and white line segments to be independent of orientation. If the difference resides in light versus dark, the results should be consonant with those of experiment 1. 
Figure 5 presents the average results of repeating experiment 1 with white tetragons.

Each of the subjects showed a pattern consistent with this average. Oddly, dark line segments did not generally require more contrast than light line segments. If the blanking phenomenon is dependent upon tetragon contrast polarity, dark line segments should have a higher threshold than light. The

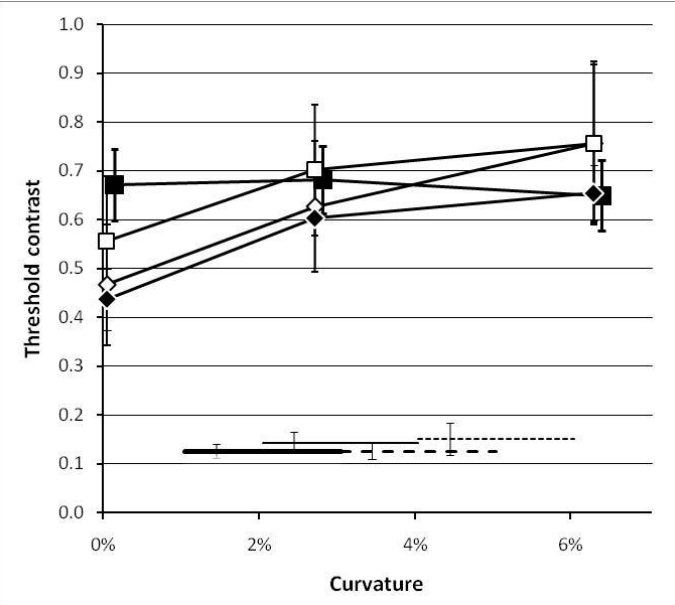

Figure 5: Results of experiment 2. White tetragons. Averages of all subjects. Conventions as in Fig. 3.

similarities of thresholds for dark and light line segments in Fig. 5 could indicate that the blanking phenomenon is weaker or absent with white tetragons (see section 4.2).

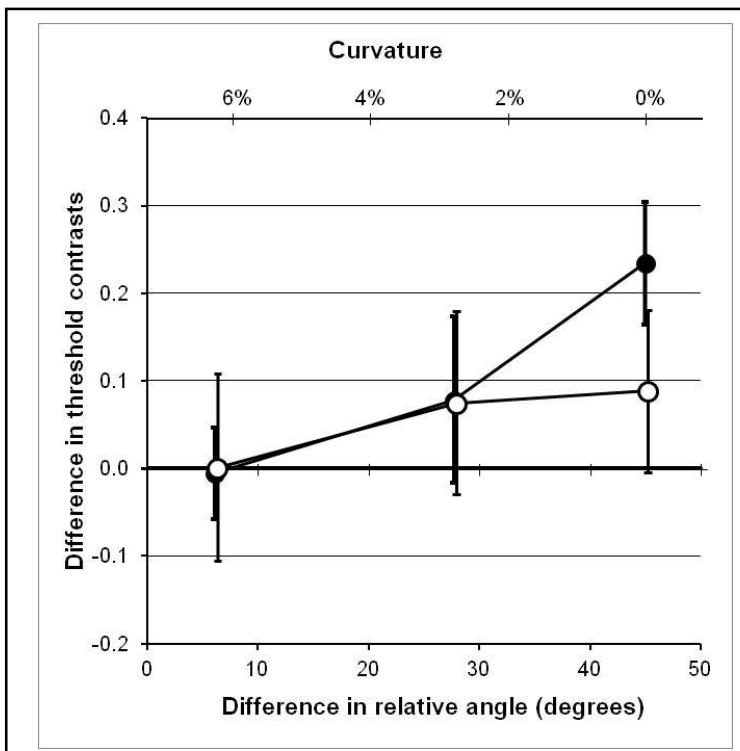

Figure 6: Data from experiment 2. Differences in contrast thresholds between cardinal and diagonal line segments versus differences in the angles between the line segments and the nearest tetragon edges. Averages of all subjects. Conventions as in Fig. 4.
Nevertheless, there is evidence for an effect of opposite contrast. Figure 5 reveals a separation of the thresholds for cardinal versus diagonal dark line segments in alleys with $0 \%$ curvature. Figure 6, comparable to Fig. 4, presents analyses of the orientation selectivity with these stimuli. As may be seen, the differences at $0 \%$ curvature are significant for dark line segments $(t[11]=7.33, p<0.001)$. The differences between dark cardinal and dark diagonal line segments with curved alleys were not significant at any other curvature, indicating 
convergence at greater curvatures. Light line segments, on the other hand, which showed an increase in threshold at greater curvatures, revealed no significant separation at any curvature.

Figure 6 also indicates that dark line segments manifest a relatively linear increase with relative angle to the nearby edges, while light line segments do not. The differences are not as clear as for black tetragons, but this may be because white tetragons are not as effective in producing blanking (note the expanded $y$-axis scale).

Although the white tetragons did not produce as dramatic a blanking effect as did the black tetragons, white tetragons nevertheless had a similar effect upon light line segments as black tetragons had upon dark line segments (i.e.: a similar obscuring effect). This may be seen in Fig. 7, which shows the means of all dark line segments in black tetragons (solid triangles) and all light line segments in white tetragons (open triangles). These are the same functions as in Figs. 2 and 4. As noted in experiment 2 , the obscured line segments in white tetragons show a greater dependence upon curvature than the dark line segments in black tetragons. For comparison, the dotted line shows the mean blanking of dark line segments in white tetragons (from Fig. 5); the blanked light line segments in black tetragons would all be at higher contrasts than the ordinate of this graph.

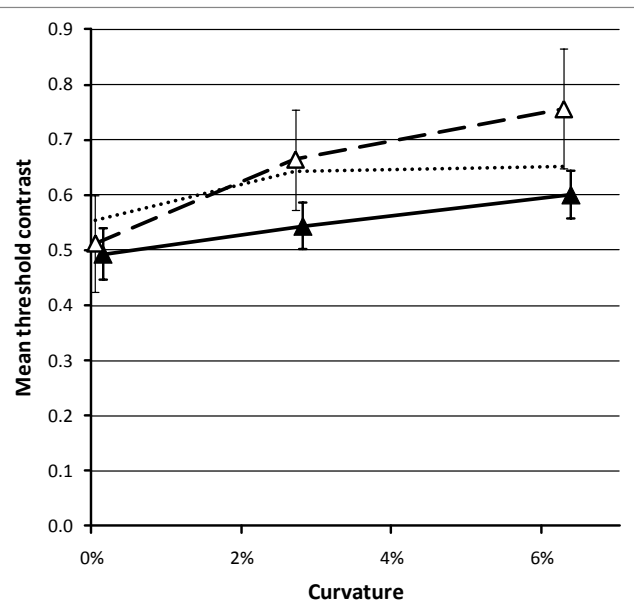

Figure 7: Data from experiments 1 and 2 for obscured (not blanked) line segments. Averages of all four line segment orientations. Open triangles and dashed line: light line segments in white tetragons. Solid triangles and solid line: dark line segments in black tetragons. Dotted line: blanked dark line segments in white tetragons. Curves are slightly shifted horizontally to segregate error bars. Error bars are $95 \%$ confidence. 


\subsection{Experiment 3: Discrimination of orientation of line segments in arrays of tetragons}

This experiment tested whether subjects could discriminate the orientations of the target line segments. Ninio and Stevens (2000) observed that their extinction illusion requires good visual acuity. The effect of orientation upon detection demonstrated that at some level subjects had the acuity necessary to discriminate orientations, at least for targets that were blanked with zero curvature. However, it is possible that orientation could not be discriminated in the presence of curvature, or at the lower contrasts of the obscured line segments that showed no orientation effect.

The display and procedures for this experiment were exactly as in experiments 1 and 2; the difference was that instead of indicating which intersection contained the line segments regardless of their orientation, subjects indicated which orientation was presented regardless of the intersection in which the target line segments appeared. Responses were made on the ring of circles in Fig. 2C. The full ring was offered so there would be no asymmetry toward a particular intersection; subjects could respond at either end of the appropriate diameter.

Each of the subjects produced results strikingly similar to those in experiments 1 and 2 . The mean results are shown in Fig. 8. Clearly, the pattern in Fig 7A closely resembles that in Fig. 3, indicating that orientation was easily discriminated with only a slight increment over the threshold for detection. As in experiment 1, cardinal orientations of light line segments required greater contrast than diagonal at $0 \%$ curvature (paired $t$-test: $t[11]=4.19, p<0.002$ ), and this difference became less (and not significant) at higher curvatures. There was no effect of orientation for dark line segments, for which the thresholds for cardinal and diagonal line segments increased in tandem as curvature increased. There was no significant difference between the thresholds for cardinal versus diagonal line segments in the absence of the tetragons. 


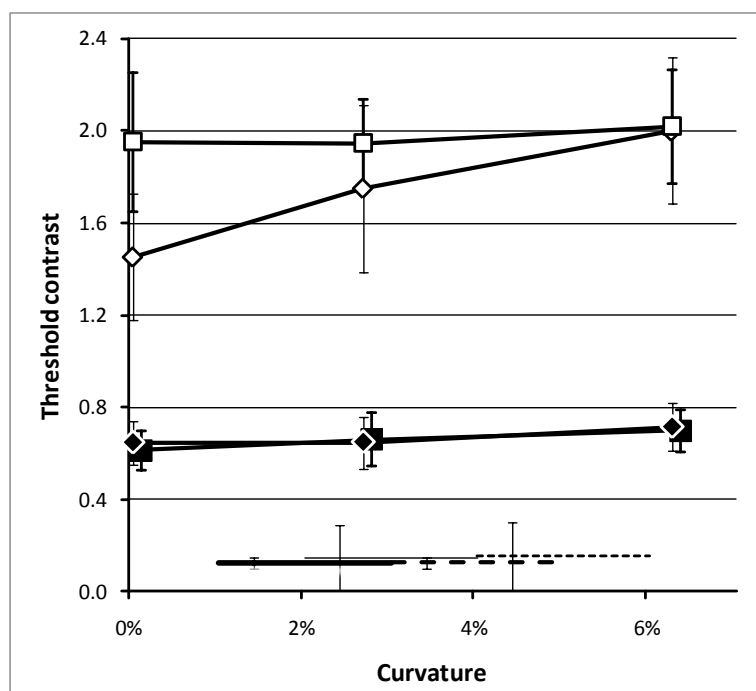

A

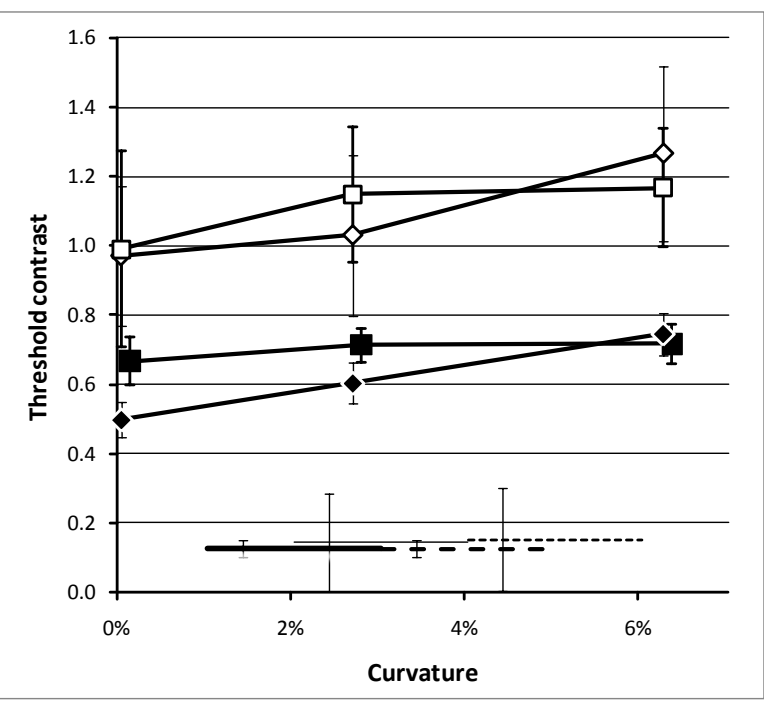

B

Figure 8: Results of discrimination of orientation; averages of all subjects. Conventions as in Fig. 3. A. Black tetragons. B. White tetragons.

Subjects were also asked to determine the orientation of the target line segments in arrays of white tetragons. These results are shown in Fig. 8B. As was the case with black tetragons, subjects discriminated orientation with only slight increases in contrast over detection thresholds. Consistent with the detection experiment, an effect of orientation was seen mainly for low curvatures and for the opposite contrast (dark) line segments (compare to Fig.4). As in experiment 2, cardinal orientations of light line segments required greater contrast than diagonal at $0 \%$ curvature (paired $t$-test: $t[11]=6.12, p<0.001$ ). Each subject displayed this general pattern.

The increase in threshold for discriminating orientation does not necessarily mean the stimulus must be consciously localized in order to discriminate its orientation, although it implies visibility may be a prerequisite. Thus, the similarity of results between experiments 1 and 2 and those of experiment 3 could rely largely on the subjects' ability to detect the stimulus as in experiments 1 and 2; note, however, that there is no difference in the abilities to discriminate orientations except for blanked stimuli with zero curvature. 


\section{Discussion}

\subsection{Blanking and complexity}

We conclude that the blanking phenomenon is sensitive to the orientation of components of the targets in the intersections relative to the orientation of the nearby edges of the tetragons comprising the array. This may be another manifestation of an observation made upon the scintillating grid that square targets (in $0^{\circ}$ curvature alleys) provide weaker scintillations than diamonds or disks (Qian et al., 2009). That is, square targets, which align well with the edges of the tetragons (squares), do poorly at engendering the illusory scintillations. However, it must be noted that the effects reported by Qian et al. used large targets relative to the alley width, and the nearness of the targets to the corners of the squares (and often occlusion of the corners) varied with target type.

As we had surmised in our earlier work, there are two distinct effects of arrays of tetragons upon detection (Levine and McAnany, 2008). Tetragons produce the blanking illusion upon targets of opposite contrast (polarity) placed in the intersections; they also increase thresholds for any target within the array, regardless of polarity. The blanking phenomenon depends upon target polarity, and, as shown here, some concordance of orientation between target and tetragons. The effect upon any targets, which we called "obscuring", depends upon the complexity of the array, but not relative orientation. Experiment 3 showed that orientation is discriminated, but has no effect upon threshold in the obscuring phenomenon. The obscuring phenomenon presumably accounts for at least some of the effects of curvature of the alleys. Both phenomena apply to targets of opposite polarity from the tetragons; only the obscuring effect of complexity applies to targets of the same polarity as the tetragons. 
The existence of two phenomena explains why the thresholds for cardinal light line segments in black tetragons exhibited little change with curvature of the alleys (this same argument applies to dark line segments in an array of white tetragons). The blanking phenomenon weakens with curvature because the cardinal line segments become less aligned with the local tetragon edges; however, the obscuring phenomenon due to increased complexity of the array increases with curvature. These opposing effects would thus tend to cancel. Diagonal light line segments, on the other hand, become better aligned with the local tetragon edges as curvature increases; the blanking phenomenon and obscuring phenomenon of complexity act in synergy to increase threshold with curvature. Dark line segments of any orientation in an array of black tetragons are not affected by blanking, so their thresholds simply increase with curvature as complexity increases (this also applies to light line segments in an array of white tetragons).

One may speculate about the origins of these phenomena. (Note that with our brief presentations, neither eye movements nor Troxler fading play a rôle.) Local orientation specificity of the blanking phenomenon implicates a mechanism in the early cortical stages, possibly V1 or V2, where cells are strongly orientation selective and receptive fields relatively spatially localized. The non-orientation selective but complexity sensitive obscuring effect may be due to "higher", less spatially specific cortical areas or their feedback upon the more primary areas (e.g.: Lamme et al., 1998; Ro et al., 2003). Ability to focus attention on the target may play a role in obscuring, as may perceptual filling-in (Araragi and Kitaoka, 2011). This could implicate parietal cortex (Lynch et al., 1977; Posner et al., 1987; Corbetta et al., 1993).

In addition, the pre-cortical component identified by the weakened effect in dichoptic presentation of tetragons and targets (McAnany and Levine, 2005) offers a contribution that 
would be neither orientation nor complexity dependent. This may well be the lateral inhibition mechanism expounded by Baumgartner (1960).

\subsection{Strength of illusion}

White tetragons often seem to have a weaker effect than black tetragons, but the difference is not usually extreme (Spillmann and Levine 1971; Spillmann, 1994). Wolfe (1984) noted that size of the tetragons (squares, in his study) in the Hermann grid is a more important parameter for white squares than for black, but also showed a slightly weaker effect for white squares of the same size as corresponding black squares (see his Figure 4). That dark line segments and light line segments were found to have similar thresholds in the presence of white tetragons may indicate that white tetragons produced only weak blanking. Were it not for the orientation preferences found for dark line segments, it might appear the white tetragons only increased thresholds (above those in the absence of tetragons) by adding complexity, the effect presumed to affect dark line segments in black tetragons.

Ninio and Stevens (2000) found arrays of white objects (triangles) to be as or perhaps even more effective than an array of black ones for the extinction illusion. This result depended upon the luminances of the alleys and whether the image was viewed on a screen or printed on paper. The difference may also depend on the fact that their targets had outlines of the opposite color, so that their white disks were outlined in black and vice versa. The outlines may have played a role in determining what could and what could not be seen, as outlines of the squares in the Hermann grid can play a significant role (Troscianko, 1982). Unlike our experiments, Ninio and Stevens also presented the displays in free-viewing; as Araragi and Kitaoka (2011) demonstrated, retinal eccentricity and temporal duration of the stimuli are important factors. Although extinction was manifested even at very short durations, the effect clearly increased 
with prolonged exposure. Araragi and Kitaoka did not consider arrays of white triangles, so it is possible that the time-courses for light and for dark targets differ. If dark targets are slower to be blanked, our short exposures might have missed further blanking evident in the prolonged exposure of free viewing. It is also worth noting that our forced-choice method is criterion-free, while free-viewing reports depend on the subject's criterion for claiming detection.

One might expect the white tetragons to be even more effective than black, since the gray of the alleys $\left(24 \mathrm{~cd} / \mathrm{m}^{2}\right)$ is physically closer to black than to white on a linear scale. Note, however, that this gray is roughly the perceptual midpoint of the display. In informal testing in which we had subjects set grays that were perceived as midway between black and white, we found a midpoint similar to the gray we used.

There are at least two possible reasons why white tetragons might be less effective at creating an illusory effect under our conditions. First, the tetragons are imposed on an otherwise neutral gray field; superposing an array of white tetragons increases overall luminance, possibly saturating some systems or changing the level of adaptation. Conversely, black tetragons lower the mean luminance of the display. The similar thresholds for the obscuring effects with the two arrays (Fig. 7) seem to argue against this possibility, but not conclusively.

There is another factor that might contribute to the relative impotence of an array of white tetragons: decremental stimuli may be somewhat more salient than incremental stimuli (Krauskopf, 1980; Bowen et al., 1989). The cited studies of incremental versus decremental stimuli relied on sawtooth changes in luminance, which may not apply here, but a more recent study by Komban et al., (2011) may be more relevant. They found that reaction times were shorter for dark targets on a black and white random noise background than were reaction times for light targets. Reaction times may not directly translate to detection thresholds, but it is 
reasonable to suggest a relationship and to propose that an effect found with black and white random patterns could also apply to regular arrays. Thus, one might expect lower thresholds for dark targets than for light targets. In an array of black tetragons, this effect would exaggerate the separation between light and dark line segments, with the lower threshold dark line segments (spared the blanking effect) being made even easier to detect because of the dark-target effect. In an array of white tetragons, thresholds for the dark line segments would be increased by blanking but mitigated by the dark-target effect; the result would be that the difference due to blanking, which might also be small because of a weaker effect of the white tetragons, would be countermanded by the dark-target effect so light and dark line segments could display similar thresholds. This asymmetry could reside in the non-orientation, non-complexity sensitive peripheral mechanism observed by McAnany and Levine (2005).

\subsection{General conclusions}

We observed a clear interaction of the orientation of light line segments with the orientation of the nearby edges of black tetragons as the alleys were curved. This implicates a local orientationspecific component to the blanking phenomenon. An orientation effect was not patent for dark line segments, further validating the distinction between the blanking phenomenon affecting light line segments and the effects of complexity that applied to dark line segments (Levine and McAnany, 2008). Of course, complexity presumably also further raises thresholds for light targets.

By using white tetragons in experiment 2, we confirmed the expectation that blanking depends on the opposite contrast of the tetragons and targets. While the blanking effect is much weaker for white tetragons (as noted in section 4.2), the orientation effect for dark line segments confirms the opposite contrast hypothesis. 
For reasons that are not clear, orientation discrimination of light line segments requires a greater increase in contrast than for dark line segments over detection thresholds in arrays of both black and white tetragons (see Fig.7). Of course, this result may depend upon the particular value of the gray comprising the alleys. The differences between black and white tetragons are worthy of further study.

We now identify four putative processes specifically affecting targets within an array of tetragons:

1. A mechanism in the pre-cortical stages (probably lateral inhibition) that is not sensitive to either orientation or complexity.

2. The blanking phenomenon, an orientation-sensitive effect that increases thresholds for opposite polarity targets within an intersection. Orientation selectivity presumably implicates early visual cortical areas.

3. The obscuring effect, in which thresholds for all targets are raised with increasing complexity (specifically achieved by increasing the curvature of the alleys). This may implicate higher cortical areas, possibly parietal cortex control of attention.

4. A possible advantage for dark targets over light targets within an array. This may minimize the apparent blanking phenomenon for white tetragons. Further study may reveal what mechanisms are responsible for the differences between arrays of white and black tetragons. 


\section{Footnote for page 5}

${ }^{1}$ Grids consist of an array of objects separated by gray "alleys". In the standard Hermann grid or scintillating grid, the objects are squares. When the alleys are curved, the four-sided objects are referred to as tetragons (a general term that includes squares and rectangles; Levine and McAnany, 2008). 


\section{REFERENCES}

Araragi Y, Kitaoka A, 2011 "Increment of the extinction illusion by long stimulation" Perception 40 608-620

Bach M, 2009 "The Hermann grid illusion: the classic textbook interpretation is obsolete" Ophthalmologe 106 913-917

Baumgartner G, 1960 “Indirekte Größenbestimmung der rezeptiven Felder der Retina beim Menschen mittels der Hermannschen Gittertäuschung" Pflügers Archiv für die gesamte Physiologie des Menschen und der Tiere 272 21-22 Cited by: Spillmann (1994)

Bowen R W, Pokorny J, Smith V C, 1989 "Sawtooth contrast sensitivity: decrements have the edge" Vision Research 29 1501-1509

Corbetta M, Miezin F M, Shulman, G L, Petersen SE, 1993 “A PET Study of Visuospatial Attention" The Journal of Neuroscience 13 1202-1226

de Lafuente V, Ruiz O, 2004 "The orientation dependence of the Hermann grid illusion" Experimental Brain Research 154 255-260

Geier J, Bernáth L, Hudák M, Séra L, 2008 "Straightness as the main factor of the Hermann grid illusion" Perception 37 651-665

Geier J, Séra L, Bernath L, 2004 "Stopping the Hermann grid illusion by simple sine distortion” ECVP 2004 Abstract 
Hering E, 1890 “Beitrag zur Lehre vom Simultankontrast” Zeitschrift fur Psychologie und Physiologie der Sinnesorgane 1 18-28 Cited by: Murch G, 1973 Visual and Auditory Perception p224 (New York: Bobbs-Merrill Company)

Howe P D L, Livingstone M S, 2007 "The Use of the Cancellation Technique to Quantify the Hermann Grid Illusion” PLoS ONE 2, e265, doi:10.1371/journal.pone.0000265

Komban S J, Alonso J-M, Zaidi Q, 2011 "Darks are processed faster than lights” Journal of Neuroscience $318654-8658$

Krauskopf J, 1980 "Discrimination and detection of changes in luminance" Vision Research. 20 $671-677$

Lamme V, Supèr H, Spekreijse H, 1998 "Feedforward, horizontal, and feedback processing in the visual cortex" Current Opinion in Neurobiology 8 529-535

Levine M W, McAnany J J, 2008 “The effects of curvature on the grid illusions” Perception 37 $171-184$

Lynch J C, Mountcastle V B, Talbot W H, Yin T C, 1977 "Parietal lobe mechanisms for directed visual attention” Journal of Neurophysiology 40 362-389

McAnany J J, Levine M W, 2004 “The blanking phenomenon: a novel form of visual disappearance" Vision Research 44 993-1001

McAnany J J, Levine M W, 2005 “A psychophysical investigation of the blanking phenomenon" Vision Research 45 193-203 
Ninio J, Stevens K A, 2000 "Variations on the Hermann grid: an extinction illusion" Perception 29 1209-1217

Posner M I, Walker J A, Friedrich F A, Rafal R D, 1987 "How do the parietal lobes direct covert attention?" Neuropsychologia 25 135-145

Qian K, Yamada Y, Kawabe T, Miura K, 2009 “The scintillating grid illusion: Influence of size, shape, and orientation of the luminance patches" Perception advance online publication doi: $10.1068 / \mathrm{p} 5943$

Ro T, Breitmeyer B, Burton P, Singhal N S, Lane D, 2003 “Feedback Contributions to Visual Awareness in Human Occipital Cortex" Current Biology 11 1038-1041

Schiller P H, Carvey C E, 2005 “The Hermann grid revisited” Perception 34 1375-1397

Spillmann L, 1994 "The Hermann grid illusion: a tool for studying human perspective field organization" Perception 23 691-708

Spillmann L, Levine J, 1971 “Contrast enhancement in a Hermann grid with variable figureground ratio" Experimental Brain Research 13 547-559

Troscianko T, 1982 "A given visual field location has a wide range of perceptive field sizes" Vision Research 22 1363-1369

Wolfe J M, 1984 “Global factors in the Hermann grid illusion” Perception 13 33-40 


\section{References index}

Araragi \& Kitaoka 2011................. 2, 19, 20

Bach, 2009 .. 2

Baumgartner 1960 2,20

Bowen, Pokorny, and Smith, 1989 21

Corbetta et al., 1993 19

de Lafuente \& Ruiz 2004 ............................ 2

Geier abstract ............................................ 2

Geier et al 2008......................................... 2

Hering 1890 ................................................. 2

Howe \& Livingstone 2007 ......................... 2

Komban, Alonso, and Zaidi 2011 ............. 21

Krauskopf, 1980...................................... 21

Lamme et al., 1998.
Levine \& McAnany 2008 .. 2, 4, 5, 7, 18, 22, 24

Lynch et al., 1977..................................... 19

McAnany \& Levine 2004 ................ 2, 4, 13

McAnany \& Levine 2005 ....... 2, 4, 5, 19, 22

Ninio \& Stevens 2000 .................... 2, 16, 20

Posner et al., 1987 ..................................... 19

Qian et al., 2009 ...................................... 18

Ro et al., 2003 ....................................... 19

Schiller and Carvey, 2005 ......................... 2

Spillmann \& Levine 1971 ......................... 20

Spillmann 1994 .................................. 20, 25

Troscianko 1982...................................... 20

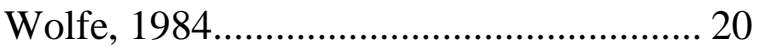

